\title{
Nano-Fabrication on the Surface of Azobenzene-Containing Copolymers by Means of Optical Near Field around Microspheres
}

\author{
Osamu Watanabe, Taiji Ikawa, Mamiko Narita, and Masaaki Tsuchimori \\ Toyota Central Research and Development Labs, \\ Nagakute, Aichi 480-1192, Japan
}

\begin{abstract}
We demonstrate a photoinduced nanofabrication using azobenzene-containing copolymers that is applicable to dimensions beyond the diffraction limit. We present a nanometer-scaled patterning induced by optical near field around arrayed polystyrene microspheres. Various azocopolymers with different glass transition temperature $(\mathrm{Tg})$ were synthesized and the depth of the nanofabrication depending on the $\mathrm{Tg}$ was examined. The dependency on the $\mathrm{Tg}$ was effected by the irradiated light power. We compared the deformation pattern on the surface with the calculated intensity distribution of the electric field around microsphere. This comparison and the polymer migration analysis show that the near-field gradient force induces the surface deformation.
\end{abstract}

Keywords: azocopolymer, optical near field, photoinduced deformation, gradient force, glass transition temperature

\section{Introduction}

In recent years, polymeric systems containing azobenzene moieties have been attracting a great deal of attention because of their potential uses in various photonic applications.[1]-[5] When exposed to light of a certain wavelength, the trans form of the azobenzene can be photoisomerized to the cis form. Cis-trans back isomerization can occur thermally or/and photochemically. This property of the azobenzene derivatives leads to a drastic change in the physical and optical properties of the polymer matrix. In this field, one of most intriguing topics is the fabrication of artificial surface relief structures in azobenzene containing polymer films by irradiation to an interference pattern, which occur due to the photoinduced mass transport process of the polymer chain.[6]-[9]

Near field optics are currently being extensively investigated from the viewpoint of nanoscale optical science and technology for superfine photolithography and high-density optical recording.[10]-[12] This is because the modification size of a conventional light source is restricted by the diffraction limit of the exposed light. We recently reported [13]-[15] that a topographical change could be induced on the surface of azobenzene containing polymer films by means of optical near field generated around polystyrene microspheres arranged on the films. The formed dents have a diameter of nearly the same or below the spheres used to form the pattern and the formation of indentations with a resolution of 20 $\mathrm{nm}$ could be achieved.[16] The indented structure thus formed was thermally stable and could be erased by heating the polymer film above glass transition temperature $(\mathrm{Tg})$.

First, in this review, we report the general description concerning the photoinduced nanofabrication process. We examined changes in the indented depth and diameter depending on the diameter of microsphere, the irradiation time and the wavelength of the irradiated light. Second, we show the formation efficiency depending on the $\mathrm{Tg}$ in the intended structure and the surface relief structure. The formation of the intended structure would be described as a result of mass transfer, which is assumed to be linked to $\mathrm{Tg}$ that is one of most important characteristic for molecular motion in polymer matrix. Various azobenzene-containing urethane copolymers with different $\mathrm{Tg}$ were synthesized. The dependency on the $\mathrm{Tg}$ was

Received March 27, 2004

Accepted May 13, 2004 
effected by the irradiated light power. Third, to explain the deformation mechanism, we compared the deformation pattern on the surface with the calculated intensity distribution of the electric field around microsphere. This comparison and the polymer migration analysis show that the near-field gradient force induces the surface deformation.

\section{Nanometer-scale patterning}

We can easily obtain various sized microspheres, from tens of nanometers to several micrometers, made from various materials such as polystyrene or silica. It is possible to place these into an ordered arrangement due to the uniformity of their diameter. We selected microspheres such as these for the near-field light source, and demonstrated a topographical nanostructure patterning technique on the surface of the azopolymer.

Nanostructured patterning was carried out as shown in Fig.la schematically. A urethane-urea copolymer containing azobenzene moieties was used, as shown in Fig. $1 \mathrm{~b}$, with a glass transition temperature of $145^{\circ} \mathrm{C}$ and an absorption maximum of $475 \mathrm{~nm}$. A film of the azocopolymer was spin-coated onto a glass substrate from a pyridine solution. Microspheres with a preselected diameter of between $2000-\mathrm{nm}$ to $28-\mathrm{nm}$ were arranged on the azopolymer. The microspheres were self-assembled into a hexagonal structure that was one monolayer thick. The sample film was irradiated from the side by $488-\mathrm{nm} \mathrm{Ar}^{+}$laser, in order to eliminate the influence of the gravity as shown in Fig.1. After irradiation, the sample was washed with water and benzene to remove the microspheres. After drying the sample, the surface structure of the polymer film was investigated using an atomic force microscope (AFM) or a scanning electron microscope (SEM).

Fig. 2 shows AFM images of the resulting polymer surface. The surface of the films prior to irradiation by $\mathrm{Ar}^{+}$laser showed no regular structural periodicity. Hexagonal structure was directly transcribed to the polymer surface as a series of indentations in the cases of the 500-nm and 100-nm microspheres. In the case of the 28-nm microspheres, an indented structure following the pattern of the microspheres was also formed, although the arrayed structure was distorted. We can confirm that these structures are induced by the optical near-field around the polystyrene microspheres, because the dimensions of the $100-\mathrm{nm}$ and 28-nm diameter spheres are beyond the diffraction limit.

Fig. 3 shows SEM images of the polymer surface, including both the indented structure and the remaining microspheres. It is confirmed that the indentaions are formed directly below the microspheres.

We explored size effect of the dents formed. Figs. 4 a

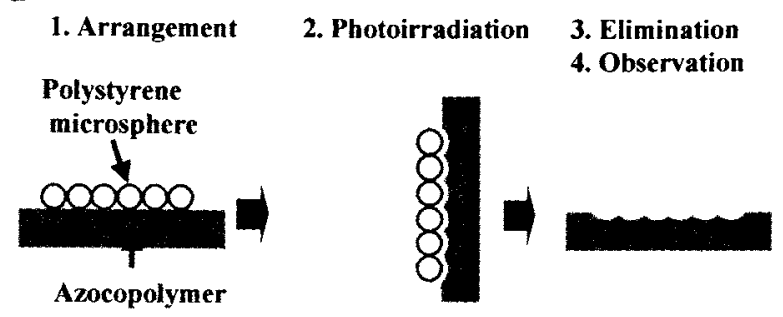

b

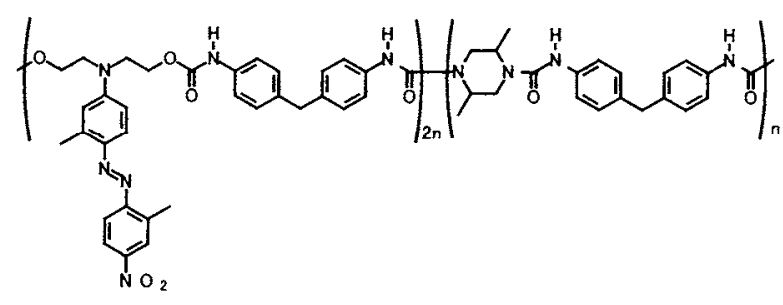

Figure 1. Schematic representation of the nanopatterning process (above) formed by using microspheres as the near-field source. Chemical structure of the azobenzene-containing urethne-urea copolymer used (below)
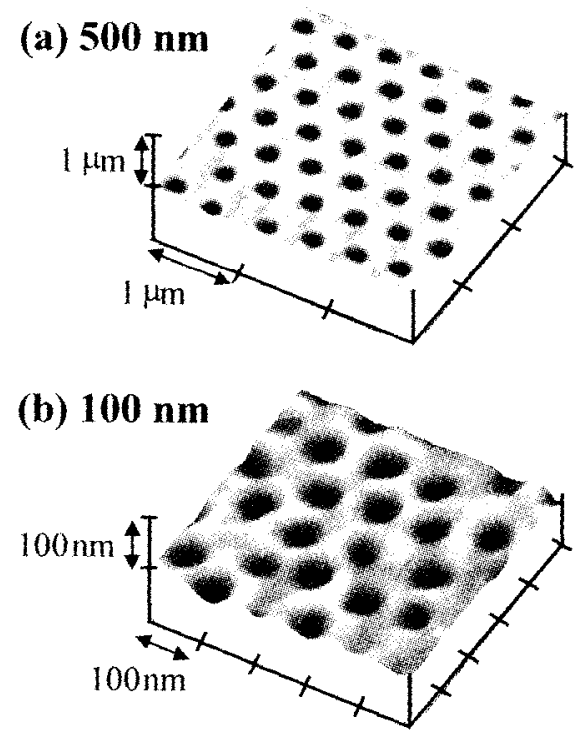

(c) $28 \mathrm{~nm}$

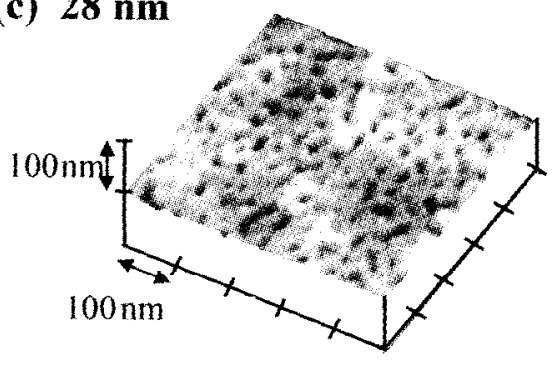

Figure 2. AFM images of nano-patterned structures formed on the azocopolymer. (a) $500 \mathrm{~nm}$ microsphers , (b) $100 \mathrm{~nm}$ microspheres, (c) $28 \mathrm{~nm}$ microspheres. 

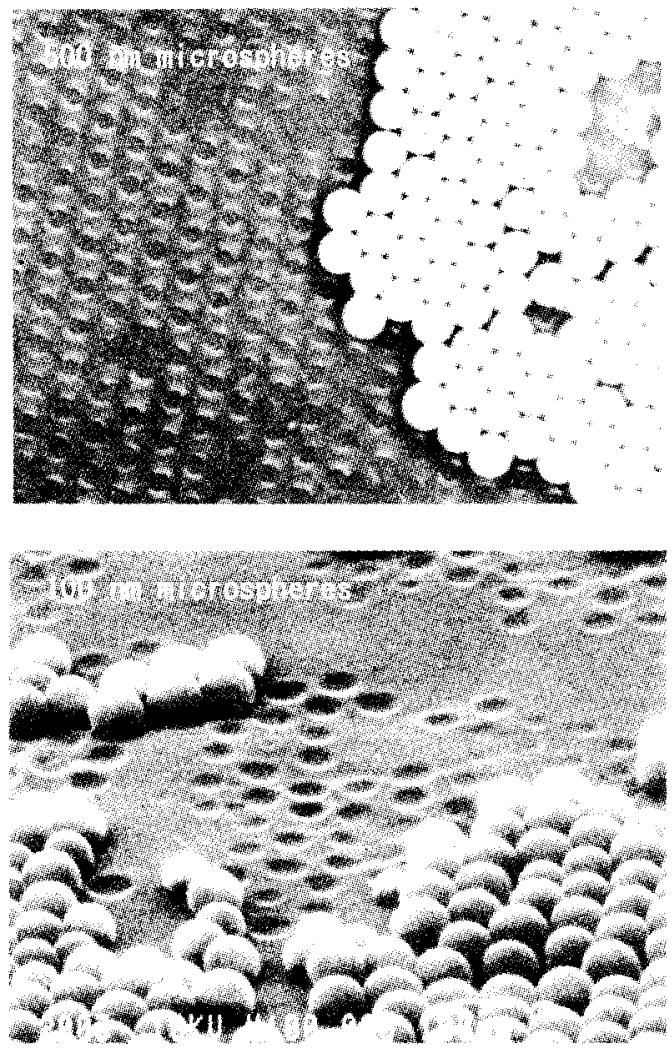

Figure 3. SEM images of the nano-patterned structure formed on the azopolymer. The microspheres partially remain.

(a) and (b) show changes in the dent depth (a) and the dent diameter (b), respectively. Both the depth and the diameter increased when we increased the size of the microspheres. For the dents formed was nearly equal to that of the microspheres used. On the other hand, the diameter of the dents formed was smaller than that of the microspheres for microspheres larger than $250 \mathrm{~nm}$. In the case of the larger microspheres, a relatively small dent would be formed due to the collection of light beneath the microsphere, and in the case of the smaller microsphere beyond the diffraction limit, dents the same size as the microsphere would be formed due to the near field around microsphere. Figs. 4(a) and (b) indicate that for every size of microsphere, the dent depth increased with increasing irradiation time, but that the diameter of the dent changed very little with time. In other words, the depth of the dents that were formed d increased with increasing irradiation time while maintaining the same diameter. Therefore, since the deformation process did not spread laterally along the plane of the microspheres, we can rule out surface tension or down-welling as factors causing the deformation.

The deformation changed depending on the wavelength of the light used for the irradiation. A deeper dent was obtained by irradiation close to the (a)

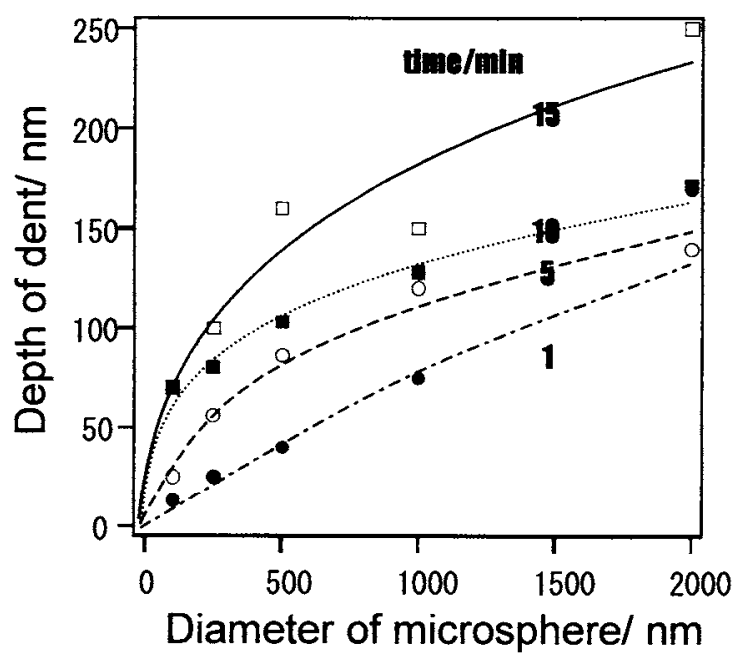

(b)

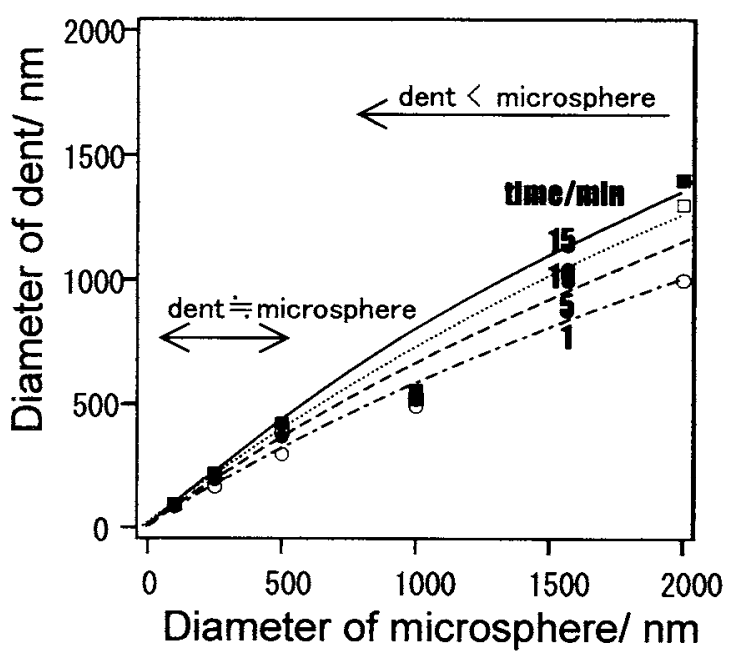

Figure 4. Changes in the dent depth (a) and the dent diameter(b) as a function of the diameter of the microspheres. Open square, solid square, open circle and solid circle show the results of the nano-patterning experiment for $15 \mathrm{~min}, 10 \mathrm{~min}, 5 \mathrm{~min}$ and $1 \mathrm{~min}$, respectively.

absorption maximum, while the depth decreased at longer wavelengths with decreasing absorption coefficient. No noticeable deformation was caused by a long irradiation at $647 \mathrm{~nm}$, where the absorption coefficient is practically zero. This demonstrates that the deformation is induced by photo-isomerization resulting from the photo-irradiation.

\section{Tg Dependency}

We compared the efficiency of deformation in various urethane copolymers, consisting of a different main chain, but containing the same azobenzene moiety and the same content. Various urethane 
Table 1. Monomer Composition, Glass Transition Temperature and Molecular Weight of the Azobenzene-containing Urethane Copolymer Used in This Work

(1)

copolymers having different $\mathrm{Tg}$ were synthesized,[17] as shown in Table 1. The nanostructure patterning was performed in the same way as before. Hexagonal structure was directly transcribed onto the copolymer surface. The indentation structure was formed on every copolymer films regardless of Tg. Fig. 5 shows changes in the dent depth as a function of $\mathrm{Tg}$. The results obtained from the 250-nm, 500-nm and 2000-nm microspheres are compared respectively. The dent depth slightly increased with increasing $\mathrm{Tg}$.

We also examined a formation of surface relief grating using the copolymers. A two-beam Mach-Zehnder interferometer setup was used for a formation of surface relief gratings on the copolymer films. By adjusting a mirror to an incident angle of $45^{\circ}$, interference beam with periodicity of $\Lambda=345 \mathrm{~nm}$ was exposed to the films. Fig. 6 shows changes in the relief depth as a function of $\mathrm{Tg}$. The relief depth also increased with increasing $\mathrm{Tg}$. Next, a formation of the surface relief grating with low-intensity exposure was performed. Fig. 7 shows change in the relief depth as a function of Tg. The relief depth obviously decreased with the $\mathrm{Tg}$. The efficiency of the relief formation changed depending on the exposure power.

These facts could be seemingly irrational phenomena. One possible explanation is that this phenomenon involves elastic recovery. A photoinduced plastization required for the nanofabrication could be caused under sufficiently high-intensity irradiation. After the irradiation, the plastization state freezes with the discontinuation of isomerization. The high $\mathrm{Tg}$ copolymer freezes faster than the low $\mathrm{Tg}$ copolymer. We presume that a degree of the elastic recovery determines the deformed depth in the sufficiently irradiated films. On the other hand, only the mobility of polymer chain could determine the amount of deformation under low-intensity irradiation.

\section{Mechanism of deformation}

Intensity of the electric field around the polystyrene microspheres was calculated using Mie theory, under the assumption that the microspheres were irradiated with a linearly polarized plane wave. 


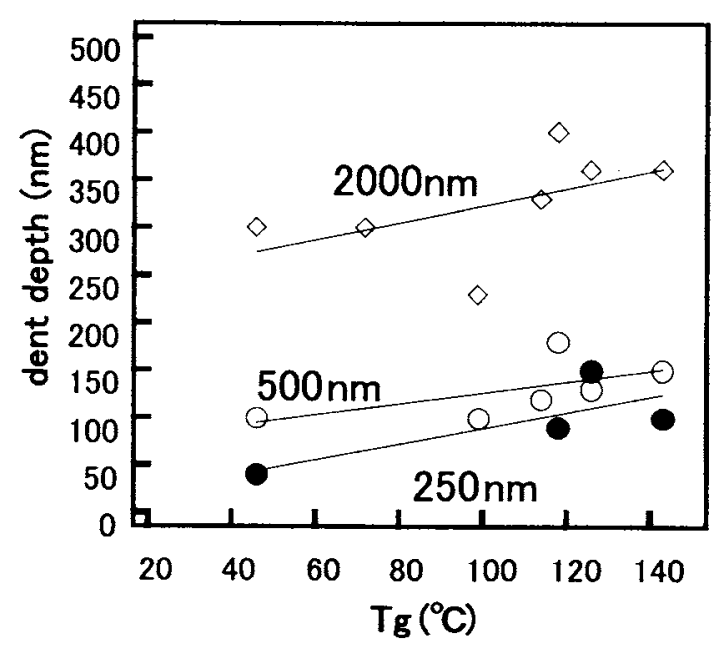

Figure 5. Changes in the dent depth as a function of $\mathrm{Tg}$. Solid circle, open circle and open rhombus show the results obtained from $250-\mathrm{nm}, 500-\mathrm{nm}$ and 2000 $\mathrm{nm}$ microspheres, respectively.

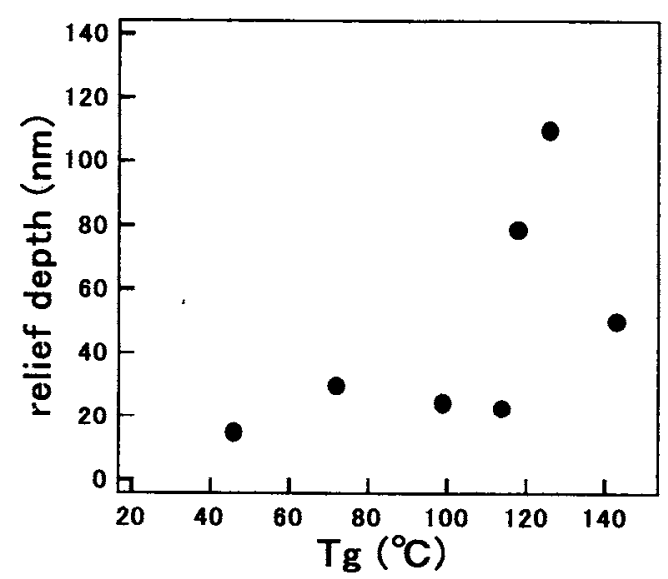

Figure 6. Changes in the relief depth as a function of Tg. Photoirradiation was carried out at $5 \mathrm{~W} / \mathrm{cm}^{2}$

[18] Calculated results are shown in Fig. 8. A relatively strong intensity was calculated in the direction of the traveling wave at the microsphere end-tip in the case of a $500-\mathrm{nm}$ microsphere, while a relatively strong intensity was calculated at the lateral part of the microsphere in the case of a $100-\mathrm{nm}$ microsphere. As described above, Fig. 3 shows that dents are formed directly below $500-\mathrm{nm}$ and $100-\mathrm{nm}$ microspheres. Comparing experimental results with the calculation, dent formation does not follow the intensity distribution of the electric field around the 100-nm microspheres, but it does for the 500-nm microspheres. In the cases of SRG formation [19] and direct beam irradiation,[20] deformation follows the intensity distribution of the electric field in the surface plane, as in the case of the $500-\mathrm{nm}$ microspheres. Therefore, we need to investigate a new mechanical force to explain deformation of the polymer surface with $100-\mathrm{nm}$

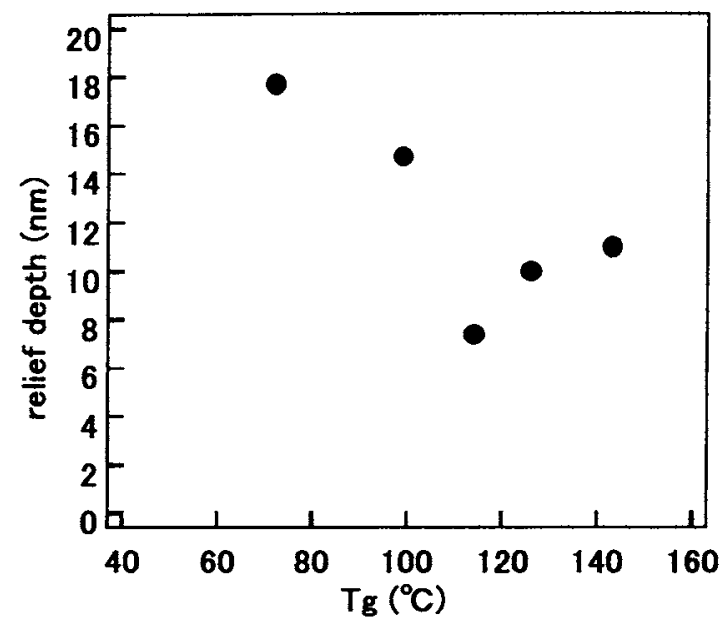

Figure 7. Changes in the relief depth as a function of Tg. Photoirradiation was carried out at $74 \mathrm{~mW} / \mathrm{cm}^{2}$

microspheres. With regard to azopolymer surface deformation in the experiment with the microspheres, we have proposed that the mechanism originates from the radiation force, including three-dimensional near-field gradient force and scattering force.

Dielectric materials in a vacuum are affected by the optical electromagnetic field. We can obtain an equation relating to dynamic force,

$$
\mathbf{F}=-\varepsilon_{0} \chi^{\prime}\left(-\nabla\left(\frac{1}{2} \mathbf{E}^{2}\right)\right)+2 \pi f \chi^{\prime \prime} \mathbf{p}_{c}
$$

where $\mathbf{F}$ is the force working on the dielectric materials, $\varepsilon_{0}$ is the electrical permitivity, $\chi$ ' and $\chi$ " are the real part and the imaginary parts, respectively, of the electrical susceptibility of the material, $\mathbf{E}$ is the electric field, $f$ is the frequency, and $p_{e}$ is the momentum of light. The first term in the equation indicates the gradient force that attracts polymer from the region with weaker electric field towards the stronger field, while the second term indicates the scattering force that acts parallel to the momentum of the photon by absorption. We estimated gradient and scattering forces around the microspheres from the equation, with the resultant forces shown schematically in Fig. 9. Deformation induced by the near-field around the polystyrene microspheres can therefore be explained successfully.

As described above, photoinduced plasticization caused by fast trans-cis-trans isomerization softens irradiated parts of the azopolymer in spite of its high glass transition temperature $(\mathrm{Tg})$. Furthermore, decrease in $\mathrm{Tg}$ near the surface has also been azopolymer makes it possible to form an indentation, even though the radiation force is very weak.

The direction of polymer migration was confirmed by tapping mode AFM (TMAFM). The phase image of the TMAFM provides a map of stiffness variations 


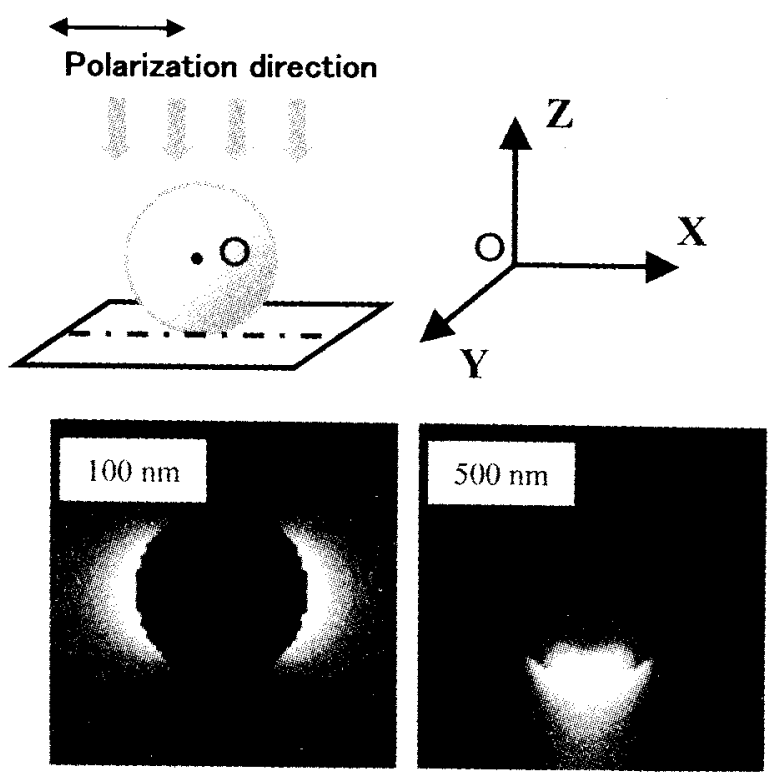

Figure 8. Calculated distribution of the optical intensity on the $\mathrm{X}-\mathrm{Z}$ plane of the polystyrene microsphere, $100 \mathrm{~nm}$ (left) and $500 \mathrm{~nm}$ (right). The bright region indicates a relatively strong intensity.

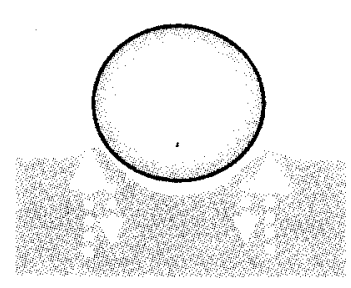

100nm microsphere

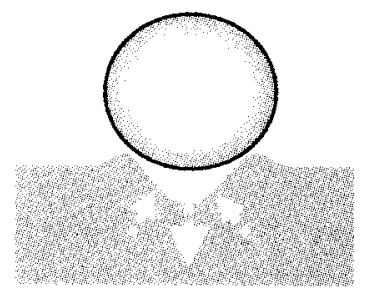

$500 \mathrm{~nm}$ microsphere
Figure 9. Schematic representation of the direction of the forces acting on the azopolymer. The up-arrow shows the gradient force and the down-arrow shows the scattering force.

a cross the surface, where a positive relative phase shift implies a stiffer region.[22] Images of the phase shift reveal that the periphery of the dent becomes harder than the interior in the case of $100-\mathrm{nm}$ microspheres, and that the interior of the dent becomes harder in the case of 500-nm microspheres. If the material becomes hardened in the direction of polymer migration, results of the phase image support a deformation mechanism as explained by radiation force.

\section{References}

1. D. M. Buland, R. D. Miller, C. A. Walsh, Chem. Rev., 94 (1994) 31

2. A. Natansohn, "Azobenzene-Containing
Materilas" Wiley-VCH Verlag Gmbh, Weinheim 3. T. Ikeda, O. Tsutsumi, Scienece, 268 (1995) 1873

4. K. Ichimura, Chem Rev., 100 (2000) 1847

5. O. Watanabe, M. Tsuchimori, A. Okada, H. Ito, Appl. Phys. Lett., 71 (1997) 750

6. P. Rochon, E. Batalla, A. Natahnsohn, Appl. Phys. Lett., 66 (1995) 1166

7. D. Y. Kim, S. K. Tripathy, L. Li, J. Kumar, Appl. Phys. Lett., 66 (1995) 1166

8. K. Sumaru, T. Yamanaka, T. Fukuda, H. Matsuda, Appl. Phys. Lett., 75 (1999) 1878

9. N. Zettsu, T. Ubukata, T. Seki, K. Ichimura, Adv. Mater., 13 (2001) 1693

10 E. Betzig, J. K. Trautman, T. D. Harris, J. S. Werner, R. L. Kostelak, Science, 251 (1991) 1468 11. B. D. Teris, H. J. Mamin, D. Rugar, W. R. Studenmund, G. S. Kino, Appl. Phys. Lett., 65 (1994) 388

12. B. Knoll, F. Keilmann, Nature 399 (1999) 134

13. Y. Kawata, C. Egami, O. Nakamura, O. Sugihara, N. Okamoto, M. Tsuchimori, O. Watanabe, Opt. Commun., 161 (1999) 6

14. M. Hasegawa, T. Ikawa, M. Tsuchimori, O. Watanabe, Y. Kawata, Macromolecules, 34 (2001) 305

15 M. Hasegawa, C. -D. Keum, O. Watanabe, Adv. Mater., 14 (2002) 1738

16. T. Ikawa, T. Mitsuoka, M. Hasegawa, M. Tsuchimori, O. Watanabe, Y. Kawata, C. Egami, O. Sugihara, N. Okamoto, J. Phys. Chem. B, 104 (2000) 9055

17. M. Narita, T. Ikawa, M. Hasegawa, M. Tsuchimori, O. Watanabe, Polym. Prepr., Jpn., 49 (2000) 3037

18. T. Ikawa, T. Mitsuoka, M. Hasegawa, M. Tsuchimori, O. Watanabe, Y. Kawata, Phys. Rev. B, 64 (2001) 195408

19. J. Kumar, L. Li, X. L. Jiang, D. -Y. Kim, T. S. Lee, S. K. Tripathy, Appl. Phys. Lett., 72 (1998) 2096

20. S. Bian, L. Li, J. Kumar, D. Y. Kim, J. Williams, S. K. Tripathy, Appl. Phys. Lett., 73 (1998) 1817

21. T. Kajiyama, K. Tanaka, A. Takahara, Macromolecules, 30 (1997) 280

22. S. N. Magnov, V. Elings, M. H. Whangbo, Surf. Sci., 375 (1997) L385 